Mahmoud I. Mahmoud, Ayman Saadany, Maged Hassan,

Hatem Essam

[1]

Chest Diseases Dept, Faculty of Medicine, Alexandria University, Alexandria, Egypt.

\title{
A rare cause of haemoptysis in a male with unprovoked deep venous thrombosis
}

Cite as: Mahmoud MI, Saadany A, Hassan M, et al. A rare cause of haemoptysis in a male with unprovoked deep venous thrombosis. Breathe 2016; 12: e12-e19.

\section{Case report}

A 29 year-old previously healthy Egyptian male complained of a 4-day vague abdominal pain for which he underwent an abdominal ultrasound. An inferior vena cava (IVC) thrombus was suspected by the sonographer who recommended undergoing an abdominal computed tomography (CT) with intravenous contrast. The IVC thrombus was confirmed by CT, but the study did not delineate any cause for the thrombus or any additional abdominal pathology. The basal chest scans revealed multiple peripheral cavitary nodules in both lower lung lobes. The patient was referred to our institution for evaluation of the pulmonary lesions. At the time, the patient only complained of an occasional cough and a small amount of mucopurulent sputum, but no chest pain. He was moderate smoker and substance abuser (tramadol and cannabis). The patient strongly denied i.v. drug abuse as well as taking any medications.

\section{Examination}

The patient's vital signs were all within normal limits and his oxygen saturation was $96 \%$ while breathing ambient air. General examination revealed oral ulcers that were tender. No needle marks could be found. Apart from right hypochondrial tenderness, cardiac, pulmonary and abdominal examination was unremarkable.

\section{Investigations}

Initial laboratory work-up showed normochromic anaemia (10.2 g. $\mathrm{dL}^{-1}$ ) and elevated C-reactive protein $\left(64 \mathrm{mg} \cdot \mathrm{dL}^{-1}\right)$, but no leukocytosis. Liver enzymes, urea and serum electrolytes were normal. A chest radiograph showed bilateral parahilar infiltrates.

Task 1

What additional blood tests are required at this stage? 


\section{Answer 1}

Given the presence of unexplained deep venous thrombosis, a thrombophilia panel is needed to explain such hypercoagulable state.

Before starting anticoagulation, a panel of tests was carried out including activity of antithrombin III, protein C and S and testing for factor V Leiden and prothrombin mutations. All test results were negative.
A dedicated chest $\mathrm{CT}$ with i.v. contrast was ordered, and showed multiple variable-sized cavitary nodules located in both upper and lower lung lobes. No adenopathy or effusion was found.

\section{Task 2}

What is the differential diagnosis for these thoracic abnormalities? 


\section{Answer 2}

Infection (specifically septic emboli), vasculitis and malignancy.

Septic emboli were considered a possible explanation for the pulmonary lesions given the history of substance abuse (from our experience, i.v. drug abuse is occasionally only admitted after a diagnosis of infective endocarditis is made). Echocardiography showed normal right-sided valves and both blood and sputum cultures were sterile.

\section{Task 3}

At this stage, which of the three possible diagnoses is more likely?
a. Hidden malignancy
b. Vasculitis
c. Infection 


\section{Answer 3}

b. Vasculitis

There was no evidence of malignancy on chest or abdominal CT. The combination of unprovoked IVC thrombosis and pulmonary nodules of undetermined aetiology directed the search to disorders associated with vasculitis.

\section{Task 4}

Which tests can be helpful for the diagnosis of vasculitis? 


\section{Answer 4}

Rheumatoid factor, anti-nuclear antibody, anti-smooth muscle antibody, anti-neutrophil cytoplasmic antibody, anti-double stranded DNA antibodies.

The rheumatoid factor was weakly positive (20 IU $\cdot \mathrm{mL}^{-1}$ ), but all other autoantibodies were negative. In addition, viral markers for HIV, hepatitis $C$ and hepatitis $B$ were all negative.

Behçet's disease, being a purely clinical diagnosis, was the only vasculitis that couldn't be excluded with negative serology. Despite the presence of oral ulcers, and the propensity of Behçet's disease to cause large venous thrombi and pulmonary infiltrates, the diagnosis could not be made due to the lack of enough clinical criteria.

\section{Task 5}

What is the next logical step?
a. Perform open lung biopsy from one of the nodules
b. Discharge the patient on anticoagulants
c. Perform flexible bronchoscopy 

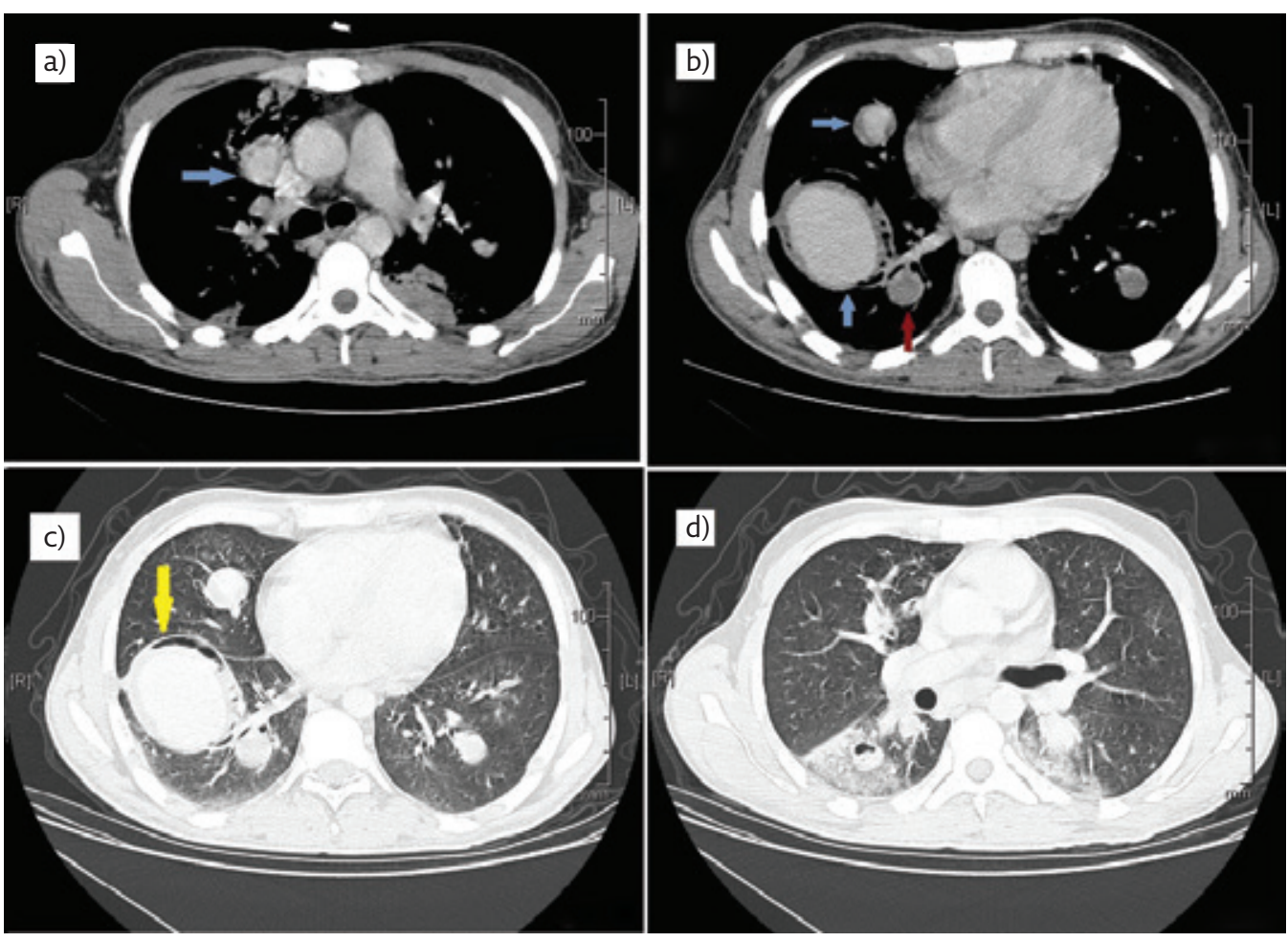

Figure 1 Chest CT of the patient.

\section{Answer 5}

a. Perform open lung biopsy from one of the nodules

A lung biopsy was, unfortunately, deferred according to patient's wishes and thus he was discharged on warfarin and a short course of antibiotics.

\section{Follow-up}

1 month later the patient appeared for follow-up in good shape, and the international normalised ratio target was reached. 3 months after the

initial complaint, the patient had two bouts of haemoptysis of $\sim 100 \mathrm{~mL}$ each time. A new chest radiograph revealed a perihilar mass lesion in the middle and lower right lung zones. The patient was admitted and a chest CT with contrast was carried out. A few hours later the patient had a massive haemoptysis upon which he was transferred to the intensive care unit, intubated and put on mechanical ventilation. Despite aggressive resuscitation and blood transfusion, the patient died an hour later.

The new chest $C T$ result arrived later.

\section{Task 6}

Describe the abnormal findings on the cuts of the chest CT shown in figure 1 . 
Answer 6

The $\mathrm{CT}$ shows multiple rounded enhancing lesions that have thick walls (blue arrows in panels $a$ and $b$ ). One of the lesions only shows rim enhancement (red arrow in panel b). The lung window (panels $\mathrm{c}$ and $\mathrm{d}$ ) shows cavitation surrounding the lesions in the right lower lobe.

These findings are consistent with multiple pulmonary artery aneurysms (PAA) at the sites of some of the previously noted nodules. Some aneurysms were occluded with thrombi (red arrow in figure 1) while others were patent with mural thrombi (blue arrows in figure 1).

Given the combination of PAA and unprovoked deep venous thrombosis in a patient who is not diagnosed with Behçet's disease, a post mortem diagnosis of Hughes-Stovin syndrome was made.

\section{Discussion}

Hughes-Stovin syndrome is an exceedingly rare disorder that is characterised by recurrent thrombophlebitis along with pulmonary and/or bronchial artery aneurysms [1]. Due to its similarity with vascular and pulmonary manifestations of Behçet's disease, it has been dubbed as "incomplete Behçet" and "cardiovascular manifestation of Behçet" [1, 2] Behçet's disease and HughesStovin syndrome are essentially the only vasculitides associated with PAA, and thus Hughes-Stovin syndrome is usually diagnosed in patients who do not meet the minimum criteria needed for Behçet's disease diagnosis. According to the International Study Group for Behçet's Disease, these minimum criteria are the presence of recurrent oral aphthous ulcers with at least two of the following symptoms: recurrent genital ulceration, uveitis and/or retinal vein affection, skin lesions, or a positive pathergy test (development of nodular rash at the site of a skin prick) [3].

Hughes-Stovin syndrome is named after two British physicians who first described four cases that had large vein thrombosis and PAA in 1959 [4], but the diagnostic label of this unique pathological entity was first used by Kopp and GREeN in 1962 [5]. Since then, $<50$ cases have been reported in the English language literature worldwide and this is why the prevalence of the disease is not known [1]. Cases have been reported from around the world. There is a strong predilection for males in the third and fourth decades of life [1].

The presented case depicts the diagnostic conundrum of a patient with pulmonary nodules and IVC thrombosis who did not match the criteria for Behçet's disease and in whom both infection and malignancy were practically excluded.

Lung nodules or cavitation develop primarily in the setting of infection or malignancy and, in certain situations, vasculitis is the culprit [6]. When pulmonary lesions are combined with large vein thrombosis that is unprovoked, malignancy and vasculitis become more likely. Without sufficient evidence of malignancy, few disorders associated with vasculitis will be able to explain the condition. A concise list of these disorders include: inflammatory bowel disease, classic autoimmune diseases and Behçet's disease [6]. While the first two categories have laboratory tests to confirm or refute their presence, Behçet's disease can only be proven by pure clinical criteria only after exclusion of other causes that have a similar presentation [7].

The patient's history of drug abuse was the reason why infection was the primary consideration. After exclusion of infection, the attention shifted to vasculitis as a plausible cause for unprovoked thrombophlebitis and pulmonary infiltrates. All possible causes were excluded after comprehensive serological testing except for Behçet's disease. The patient had oral ulcers, pulmonary infiltrates and IVC thrombus, which can all fit in the Behçet's disease spectrum, but after rheumatology consultation the diagnosis was deemed immature. In hindsight we believe this was not the best decision. Uzun [8] has argued that clinicians need not expect the full picture of Behçet's disease to be evident at presentation and that some manifestations of the disease take years to develop. This way, patients would not be denied possible treatments. It was unfortunate lung biopsy was put off at this stage, as we feel it might have pointed to an inflammatory cause of the manifestation and catalysed a more aggressive therapeutic approach.

The appearance of PAA later in the course made Hughes-Stovin syndrome, a well-recognised forme fruste of Behçet's disease, the perfect fit. PAA have been reported to occur on top of pulmonary artery occlusion [9]. The temporal gap between the development of PAA and presentation as pulmonary nodules is probably unique to this case in comparison to the natural history of previously reported Hughes-Stovin syndrome cases. We think that these cavitary nodules may have been infarcted areas secondary to small occlusions in the pulmonary circulation and were the precursors for the development of the PAA. In the original account of the condition by Hughes and Stovin, structural changes in the bronchial arteries were suggested to impair nutrition to the pulmonary arteries, which led to the damage of their walls and their dilatation $[4,10]$. This argument is still valid and can explain the sequence of events.

The reported patient died of massive haemoptysis, which has been the fate of $50 \%$ of the reported cases [1]. The cause of death is either pulmonary artery thrombosis or ruptured aneurysm [1]. It should be noted that aneurysms in HughesStovin syndrome can be single or multiple and can occur on both the arterial and venous circulation, with the latter usually being of little clinical significance [1]. Occlusions are also encountered in the venous and arterial sides, and the deep veins 
of the lower limbs are the commonest sites [11]. Cerebral venous sinus thrombosis can occur causing grave neurological manifestations $[4,11]$. An important point to be made is that pulmonary arterial occlusions are usually due to in situ thrombosis rather than embolisation from deep venous thrombosis, as it has been noted that thrombin is tightly adherent to the walls of these veins [9, $11,12]$. One of the peculiar radiological signs that point to an inflammatory cause of pulmonary thrombosis is the finding of a rim of enhancement adjacent to the filling defect (figure 1a) [9].

Had this pattern of the natural history of HughesStovin syndrome been recognised earlier, our patient would have had several therapeutic options. Just like Behçet's disease, immunosuppressive therapy (mainly cyclophosphamide) and corticosteroids are the cornerstones of therapy [7]. Several reports have shown regression or resolution of PAA after medical treatment $[13,14]$. The question of anticoagulation is highly controversial, particularly in patients with haemoptysis. Its use can only be advocated in cases of massive pulmonary thrombosis or intra-cardiac thrombi, with the recognised risk of precipitating fatal haemorrhage [1]. In patients who have a single or few PAA or who present with massive haemoptysis secondary to aneurysm rupture, surgery in the form of lobectomy or pneumonectomy is sometimes attempted [12]. Given the multiplicity of lesions in most cases and the bad general status of the patients at presentation, a more conservative approach in the form of percutaneous embolisation of the bleeding aneurysm is more commonly applied with some success [15].

\section{Conclusion}

In conclusion, both Behçet's disease and HughesStovin syndrome may manifest with cavitary nodules because they belong to the same continuum. Despite having considerable fatality, timely diagnosis under either of the labels should prompt satisfactory management.

\section{Conflict of interest}

None declared.

\section{References}

1. Khalid U, Saleem T. Hughes-Stovin syndrome. Orphanet J Rare Dis 2011; 6: 15

2. Erkan D, Yazici Y, Sanders A, et al. Is Hughes-Stovin syndrome Behçet's disease? Clin Exp Rheumatol 2004; 22: Suppl., S64-S68.

3. Criteria for diagnosis of Behçet's disease. International Study Group for Behçet's Disease. Lancet 1990; 335: 1078-1080.

4. Hughes JP, Stovin PG. Segmental pulmonary artery aneurysms with peripheral venous thrombosis. $\mathrm{Br} J$ Dis Chest 1959; 53: 19-27.

5. Kopp WL, Green RA. Pulmonary artery aneurysms with recurrent thrombophlebitis. The "Hughes-Stovin syndrome". Ann Intern Med 1962; 56: 105-114.

6. Brown KK. Pulmonary vasculitis. Proc Am Thorac Soc 2006; 3 : 48-57.

7. Uzun O, Akpolat T, Erkan L. Pulmonary vasculitis in Behçet disease: a cumulative analysis. Chest 2005; 127: 2243-2253.

8. Uzun O. Pulmonary involvement in Behçet's disease and Takayasu's arteritis. In: Cordier J-F, ed. Orphan Lung Disease (ERS Monograph). Sheffield, European Respiratory Society, 2011; pp. 32-45.
9. Ketchum ES, Zamanian RT, Fleischmann D. CT angiography of pulmonary artery aneurysms in Hughes-Stovin syndrome. AJR Am J Roentgenol 2005; 185: 330-332.

10. Charlton RW, Du Plessis LA. Multiple pulmonary artery aneurysms. Thorax 1961; 16: 364-371.

11. Emad Y, Ragab Y, Shawki Ael-H, et al. Hughes-Stovin syndrome: is it incomplete Behçet's? Report of two cases and review of the literature. Clin Rheumatol 2007; 26: 1993-1996.

12. Durieux P, Bletry O, Huchon G, et al. Multiple pulmonary arterial aneurysms in Behcet's disease and HughesStovin syndrome. Am J Med 1981; 71: 736-741.

13. Lee J, Noh J-W, Hwang JW, et al. Successful cyclophosphamide therapy with complete resolution of pulmonary artery aneurysm in Hughes-Stovin syndrome patient. Clin Rheumatol 2008; 27: 1455-1458.

14. Schreiber BE, Noor N, Juli CF, et al. Resolution of Behçet's syndrome associated pulmonary arterial aneurysms with infliximab. Semin Arthritis Rheum 2011; 41: 482-487.

15. Herb S, Hetzel M, Hetzel J, et al. An unusual case of Hughes-Stovin syndrome. Eur Respir J 1998; 11: 11911193. 